BIOKEMISTRI 18(2):127-132 (December 2006)

Available online at http://www.bioline.org.br/bk and at http://www.ajol.info/journals/biokem

Printed in Nigeria

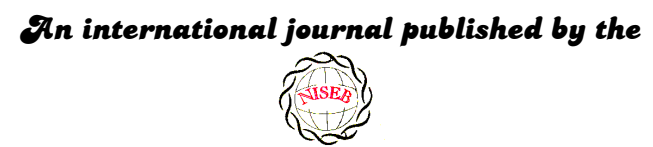

OVigerian Society for \&̊xperimental Siology

\title{
Prostate disorders in an apparently normal Nigerian population 1: Prevalence
}

\author{
Lawrence U.S. EZEANYIKA* ${ }^{1}$, Chukwunonso E.C.C. EJIKE ${ }^{1}$, Onyechi OBIDOA ${ }^{1}$ and \\ Sunday O. ELOM ${ }^{2}$ \\ ${ }^{1}$ Department of Biochemistry, University of Nigeria, Nsukka, Nigeria \\ ${ }^{2}$ Department of Medical Biochemistry, Ebonyi State University, Abakaliki, Nigeria
}

Received 15 June 2006

MS/No BKM/2006/015, ( 2006 Nigerian Society for Experimental Biology. All rights reserved.

\begin{abstract}
Benign prostate hyperplasia $(\mathrm{BPH})$ constitutes a lot of health burden for adult males. Prevalence statistics are well documented in the developed world, but not so well documented in Africa, especially in Nigeria. This study was therefore carried out to provide information on the prevalence of BPH in this locality. Adult males (aged forty years or older) who reside in Nsukka, Enugu State Nigeria, who had no apparent symptoms of ill health, were used for the study. The International Prostate Symptom Score (IPSS) index was used to establish the presence of BPH, respondents' choice of most troublesome symptom and respondents' perception of their quality of life. The results show that $25.35 \%$ of the studied population had symptoms suggestive of BPH. The severity of symptoms was seen to increase with age. The respondents regarded Nocturia as the most troublesome symptom of $\mathrm{BPH}$, while storage symptoms were seen to be more troublesome than voiding symptoms. Also $18.91 \%$ of the studied population had poor quality of life, while $81.08 \%$ were satisfied with their quality of life. The prevalence of $\mathrm{BPH}$ in the studied population shows that one in four apparently normal men have BPH. This is comparable to figures from the developed world. Sadly the awareness of the problem in Nigeria is not comparable to that in the developed world. This calls for a concerted effort to reverse this trend. This would check the devastating effect of BPH on the quality of life of men and thereby enhance productivity.
\end{abstract}

Keywords: Benign prostate hyperplasia (BPH), prevalence, population

*Author to whom correspondence should be addressed.

E-mail: lusezeanyika@yahoo.com , Tel: +234-803 7740067 


\section{INTRODUCTION}

Urinary problems in men are often caused by prostate disorders - prostate cancer, benign prostate hyperplasia $(\mathrm{BPH})$ and prostatitis. The most common of these disorders is BPH. This condition is characterized by an excessive increase in the number of cells in the prostate. Alterations in the size of the prostate could affect the bladder or constrict the urethra, resulting in lower urinary tract symptoms that characterize the clinical setting of $\mathrm{BPH}^{1}$.

Lower urinary tract symptoms are divided into voiding or obstructive symptoms and storage or irritative symptoms ${ }^{2}$. Voiding symptoms result from direct urinary flow obstruction while storage symptoms appear to be due to secondary bladder dysfunction. Dynamic obstruction secondary to contraction of the smooth muscles of the prostate, urethra and bladder neck could also cause obstructive symptoms. Bladder wall hypertrophy and collagen deposition in the bladder appear to cause storage symptoms ${ }^{3}$.

The voiding/obstructive symptoms are incomplete emptying, intermittency, weak stream and straining/hesitancy. The storage/irritative symptoms are urinary frequency during the day, urgency/urge incontinence and nocturia ${ }^{2}$. These symptoms of $\mathrm{BPH}$ affect the quality of life (QOL) of patients adversely. The degree of discomfort and the particular symptom regarded as most troublesome differ from patient to patient.

Several methods are available for the screening and diagnosis of BPH. However, the International Prostate Symptom Score (IPSS) index, a modification of the American Urological Association (AUA) symptom index ${ }^{4,5}$ appears to be the method requiring little skill and no laboratory equipment. This makes the IPSS index a particularly useful screening tool in the third world where equipment for tests like uroflowmetry, ultrasonography, etc, may not be readily available. The IPSS index consists of questions on the symptoms of $\mathrm{BPH}$, most bothersome symptom and the candidate's perception of his quality of life.

The prevalence of BPH is well documented in other parts of the world, except in Africa ${ }^{6,7}$. This work was therefore carried out to provide information on the prevalence of BPH using the IPSS questionnaire.

\section{MATERIALS AND METHODS}

\section{Subjects}

Adult males aged forty years and above who reside in Nsukka, Enugu state Nigeria, who had no apparent symptoms of ill health were used for the study.

\section{Methods}

One thousand copies of the IPSS index were distributed to the subjects. The subjects were allowed to study the questionnaire and ask questions about the study where necessary. The aim of the study was explained to participants to encourage participation. Those who consented completed the questionnaires. Privacy and secrecy were maintained throughout the duration of the exercise. No honoraria were paid to participants.

The completed and returned questionnaires were analysed to generate the results. The tally system was used in counting and was done in duplicates. Total scores were obtained by adding up the subjects score on each question. With the total scores, candidates were grouped into three categories - mild urethral obstruction (0-7), moderate urethral obstruction (8-19) and severe urethral obstruction (20-35) ${ }^{4}$. Subjects were also grouped according to the symptom they considered most troublesome. From the grouping, those who regarded voiding or obstructive symptoms as the most troublesome were separated from those who saw their most troublesome symptoms in storage or irritative symptoms. Finally, based on the subjects' perception of their QOL, they were divided into three groups - satisfied, mixed (satisfied/dissatisfied), and dissatisfied.

\section{RESULTS}

Of the 1000 questionnaires distributed, 817 were recovered from respondents, giving a recovery rate of $81.7 \%$. Twenty four (24) of these returned questionnaires were invalidated due to errors that rendered them unacceptable or incomprehensible. Effectively, 793 
questionnaires were accepted and used as valid, representing $79.3 \%$ of the questionnaires distributed and $97.06 \%$ of the questionnaires recovered from respondents. Of this number, only 371 subjects responded to the question on the most troublesome symptom, representing $46.8 \%$ of the questionnaires accepted as valid, $45.4 \%$ of returned questionnaires and $37.1 \%$ of distributed questionnaires. Data from the study are presented in Table 1 .

Table 1: Symptom scores, number of individuals affected and their percentages.

\begin{tabular}{|c|c|c|c|c|c|c|c|c|c|c|}
\hline & \multicolumn{2}{|c|}{$40-49$} & \multicolumn{2}{|c|}{$50-59$} & \multicolumn{2}{|c|}{$60-69$} & \multicolumn{2}{|c|}{$70+$} & \multicolumn{2}{|c|}{ Together } \\
\hline & $\mathrm{N}$ & $\%$ & $\mathrm{~N}$ & $\%$ & $\mathrm{~N}$ & $\%$ & $\mathrm{~N}$ & $\%$ & $\mathrm{~N}$ & $\%$ \\
\hline Mild & 288 & 83.00 & 245 & 82.50 & 34 & 34.70 & 25 & 49.02 & 592 & 74.65 \\
\hline Moderate & 58 & 16.71 & 49 & 16.50 & 61 & 62.24 & 19 & 37.25 & 187 & 23.58 \\
\hline Severe & 1 & 0.29 & 3 & 1.01 & 3 & 3.06 & 7 & 13.73 & 14 & 1.77 \\
\hline Total & 347 & 43.76 & 297 & 37.45 & 98 & 12.36 & 51 & 6.43 & 793 & 100 \\
\hline
\end{tabular}

$N=$ number of individuals affected.

Table 2: Symptom scores, number of individuals affected and nature of symptom

\begin{tabular}{lllllllllll}
\hline & $\mathrm{N}$ & $\mathbf{1}$ & $\mathbf{2}$ & $\mathbf{3}$ & $\mathbf{4}$ & $\mathbf{5}$ & $\mathbf{6}$ & $\mathbf{7}$ & $\mathbf{V S}$ & SS \\
\hline Mild & 219 & 11 & 22 & 22 & 43 & - & - & 121 & 33 & 186 \\
$\mathrm{n}=592$ & $(42.8 \%)$ & $(5 \%)$ & $(10 \%)$ & $(10 \%)$ & $(19.6 \%)$ & & & $(55.3 \%)$ & $(15.1 \%)$ & $(84.9 \%)$ \\
Moderate & 139 & - & - & 9 & 53 & 5 & 27 & 45 & 41 & 98 \\
$\mathrm{n}=187$ & $(74.3 \%)$ & & & $(6.5 \%)$ & $(38.1 \%)$ & $(3.6 \%)$ & $(19.4 \%)$ & $(32.4 \%)$ & $(29.5 \%)$ & $(70.5 \%)$ \\
Severe & 13 & - & - & - & 5 & - & 5 & 3 & 5 & 8 \\
$\mathrm{n}=14$ & $(92.9 \%)$ & & & & $(38.5 \%)$ & & $(38.5 \%)$ & $(23.1 \%)$ & $(38.5 \%)$ & $(61.5 \%)$ \\
\hline Together & 371 & 11 & 22 & 31 & 101 & 5 & 32 & 169 & 79 & 292 \\
$\mathrm{n}=793$ & $(46.8 \%)$ & $(3 \%)$ & $(5.9 \%)$ & $(8.4 \%)$ & $(27.2 \%)$ & $(1.3 \%)$ & $(8.6 \%)$ & $(45.6 \%)$ & $(21.3 \%)$ & $(78.7 \%)$ \\
\hline
\end{tabular}

Table 1 shows that there was a progressive increase in the percentage of those with severe symptoms, suggestive of $\mathrm{BPH}$ as the age range increased from $40-49$ to $70+$. More than $80 \%$ of those between the ages of 40 and 59 had mild symptoms while more than $50 \%$ of those older than 60 years had moderate and severe symptoms (when taken together). Summarily, $74.65 \%$ of the studied population had mild symptoms while $23.58 \%$ and $1.77 \%$ of the population had moderate and severe symptoms respectively.

Table 2 shows that $55.3 \%$ of those with mild symptoms felt nocturia (7) was their most troublesome symptom while none of them had problems of weak stream (5) and straining (6). For those with moderate symptoms, urgency (4) was the most troublesome symptom for $38.1 \%$ of the population. Nocturia (7) was the next most troublesome symptom for this group. Nobody in this group saw incomplete emptying (1) and urinary frequency (2) as the most troublesome symptom. Urgency (4) and straining (6) were regarded as the most troublesome symptoms by $38.5 \%$ (in each case) of those with severe symptoms. Only $23.1 \%$ of the population felt nocturia (7) was their most troublesome symptom while nobody in the group regarded incomplete emptying (1), frequency (2), intermittency (3), and weak stream (5), as the most troublesome symptom.

The percentage of those with voiding symptoms as their most troublesome symptom increased from mild to severe groups. However, the percentage of those who regarded storage symptoms as their most troublesome symptoms dropped as the symptoms increased from mild to severe. 
As seen in Table 3, $81.08 \%$ of the sampled population felt satisfied with their quality of life and would be glad to have it so for the rest of their lives. However, $6.05 \%$ and $12.86 \%$ of the same population felt dissatisfied and both satisfied and dissatisfied (mixed) with their quality of life respectively. That is $18.91 \%$ of the population were not entirely satisfied with their quality of life. The percentage of those who felt satisfied dropped as the age range increased.

From Table 4, $45.2 \%$ of those who felt satisfied with their quality of life saw nocturia (7) as their most troublesome symptom, while $28.6 \%$ felt urgency (4) was their most troublesome symptom. A similar trend was seen for those who felt both satisfied and dissatisfied (mixed). More than half $(53.5 \%)$ of those who felt dissatisfied regarded nocturia (7) as the most troublesome symptom. Urgency (4) and straining (6) were regarded as the most troublesome symptoms by $23.3 \%$ each, of the dissatisfied population. Incomplete emptying (1), urinary frequency (2), intermittency (3) and weak stream (5) were not ticked as the most troublesome symptom by anyone who felt dissatisfied with his quality of life. The percentage of those who had voiding symptoms and storage symptoms as their most troublesome symptoms were about the same for the three groups.

Table 3: Respondents' perception of their quality of life

\begin{tabular}{|c|c|c|c|c|c|c|c|c|c|c|}
\hline & \multicolumn{2}{|c|}{$40-49$} & \multicolumn{2}{|c|}{$50-59$} & \multicolumn{2}{|c|}{$60-69$} & \multicolumn{2}{|c|}{$70+$} & \multicolumn{2}{|c|}{ Together } \\
\hline & $\mathrm{N}$ & $\%$ & $\mathrm{~N}$ & $\%$ & $\mathrm{~N}$ & $\%$ & $\mathrm{~N}$ & $\%$ & $\mathrm{~N}$ & $\%$ \\
\hline Satisfied & 330 & 95.10 & 253 & 85.19 & 48 & 48.98 & 12 & 25.53 & 643 & 81.08 \\
\hline Mixed & 12 & 3.46 & 30 & 10.10 & 41 & 41.84 & 19 & 37.25 & 102 & 12.86 \\
\hline Dissatisfied & 5 & 1.44 & 14 & 4.71 & 9 & 9.18 & 20 & 39.22 & 48 & 6.05 \\
\hline Total & 347 & 100 & 297 & 100 & 98 & 100 & 51 & 100 & 793 & 100 \\
\hline
\end{tabular}

$N=$ number of people.

Table 4: Quality of living of respondents and nature of symptom

\begin{tabular}{lllllllllll}
\hline & $\mathrm{N}$ & $\mathbf{1}$ & $\mathbf{2}$ & $\mathbf{3}$ & $\mathbf{4}$ & $\mathbf{5}$ & $\mathbf{6}$ & $\mathbf{7}$ & VS & SS \\
\hline Satisfied & 248 & 10 & 13 & 25 & 71 & 4 & 13 & 112 & 52 & 196 \\
$\mathrm{n}=643$ & $(38.6 \%)$ & $(4 \%)$ & $(5.2 \%)$ & $(10.1 \%)$ & $(28.6 \%)$ & $(1.6 \%)$ & $(5.2 \%)$ & $(45.2 \%)$ & $(21 \%)$ & $(79 \%)$ \\
Mixed & 80 & 1 & 9 & 6 & 20 & 1 & 9 & 34 & 17 & 63 \\
$\mathrm{n}=102$ & $(78.4 \%)$ & $(1.3 \%)$ & $(11.3 \%)$ & $(7.5 \%)$ & $(25 \%)$ & $(1.3 \%)$ & $(11.3 \%)$ & $(42.5 \%)$ & $(21.3 \%)$ & $(78.7 \%)$ \\
Dissatisfied & 43 & - & - & - & 10 & - & 10 & 23 & 10 & 33 \\
$\mathrm{n}=48$ & $(89.6)$ & & & & $(23.3 \%)$ & & $(23.3 \%)$ & $(53.5 \%)$ & $(23.3 \%)$ & $(76.7 \%)$ \\
\hline Together & 371 & 11 & 22 & 31 & 101 & 5 & 32 & 169 & 79 & 292 \\
$\mathrm{n}=793$ & $(46.8 \%)$ & $(3 \%)$ & $(5.9 \%)$ & $(8.4 \%)$ & $(27.2 \%)$ & $(1.3 \%)$ & $(8.6 \%)$ & $(45.6 \%)$ & $(21.3 \%)$ & $(78.7 \%)$ \\
\hline
\end{tabular}

$N=$ number of people, the numbers $1,2,3,4,5,6$, and 7 , represent the following symptoms respectively - incomplete emptying, frequency, intermittency, urgency, weak stream, straining, and nocturia. VS and SS stand for voiding symptoms and storage symptoms respectively.

Table 5: Age of Respondents and Nature of Symptom

\begin{tabular}{lllllllllll}
\hline & $\mathrm{N}$ & $\mathbf{1}$ & $\mathbf{2}$ & $\mathbf{3}$ & $\mathbf{4}$ & $\mathbf{5}$ & $\mathbf{6}$ & $\mathbf{7}$ & VS & SS \\
\hline $\mathbf{4 0 - 4 9}$ yrs & 148 & - & - & - & 50 & - & 7 & 91 & 7 & 141 \\
$\mathrm{n}=347$ & $(42.7 \%)$ & & & & $(33.8 \%)$ & & $(4.7 \%)$ & $(61.5 \%)$ & $(4.7 \%)$ & $(95.3 \%)$ \\
$\mathbf{5 0 - 5 9}$ yrs & 115 & 11 & 16 & 18 & 21 & - & 5 & 44 & 34 & 81 \\
$\mathrm{n}=297$ & $(38.7 \%)$ & $(9.6 \%)$ & $(13.9 \%)$ & $(15.7 \%)$ & $(18.3 \%)$ & & $(4.3 \%)$ & $(38.3 \%)$ & $(29.6 \%)$ & $(70.4 \%)$ \\
$\mathbf{6 0 - 6 9}$ yrs & 55 & - & - & 6 & 22 & 3 & 12 & 22 & 21 & 44 \\
$\mathrm{n}=98$ & $(56.1 \%)$ & & & $(9.2 \%)$ & $(33.8 \%)$ & $(4.6 \%)$ & $(18.5 \%)$ & $(33.8 \%)$ & $(32.3 \%)$ & $(67.7 \%)$ \\
$\mathbf{7 0 +}$ yrs & 43 & - & 6 & 7 & 8 & 2 & 8 & 12 & 17 & 26 \\
$\mathrm{n}=51$ & $(84.3 \%)$ & & $(14 \%)$ & $(16.3 \%)$ & $(18.6 \%)$ & $(4.7 \%)$ & $(18.6 \%)$ & $(27.9 \%)$ & $(39.5 \%)$ & $(60.5 \%)$ \\
\hline Together & 371 & 11 & 22 & 31 & 101 & 5 & 32 & 169 & 79 & 292 \\
$\mathrm{~N}=793$ & $(46.8 \%)$ & $(3 \%)$ & $(5.9 \%)$ & $(8.4 \%)$ & $(27.2 \%)$ & $(1.3 \%)$ & $(8.6 \%)$ & $(45.6 \%)$ & $(21.3 \%)$ & $(78.7 \%)$ \\
\hline
\end{tabular}

$N=$ number of people, the numbers 1, 2, 3, 4, 5, 6, and 7, represent the following symptoms respectively - incomplete emptying, frequency, intermittency, urgency, weak stream, straining, and nocturia. VS and SS stand for voiding symptoms and storage symptoms respectively. 
Table 5 shows that $61.5 \%$ of those aged $40-49$ years felt nocturia (7) was their most troublesome symptom. A total of $33.8 \%$ of the same population felt urgency (4) was the most troublesome symptom while only $4.7 \%$ of that population felt straining (6) was the most troublesome symptom. The other symptoms were not seen as the most troublesome by anyone in this age range. For those aged 50-59, only weak stream (5) is not seen as a problem by anyone. As much as $38.3 \%$ of the population of this age range regarded nocturia (7) as their most troublesome symptom. Urgency (4) and nocturia (7) each was regarded by $33.8 \%$ of those aged 60-69 as their most troublesome symptom. Nobody in this age bracket regarded incomplete emptying (1) and urinary frequency (2) as the most troublesome symptom. For the age range $70+, 27.9 \%$ of the population felt that nocturia (7) was the most troublesome symptom while nobody regarded incomplete emptying as the most troublesome symptom. The percentage of those who regarded voiding symptoms as their most troublesome symptoms increased as the age range increased. The reverse is seen to be the case with those who regarded storage symptoms as the most troublesome.

From all the tables, it is seen that $78.7 \%$ of the entire population that responded to the question on most troublesome symptom felt storage symptoms were the most troublesome symptoms while $21.3 \%$ felt voiding symptoms were the most troublesome symptoms. A majority (45.6\%) of the population regarded nocturia (7) as the most troublesome symptom while only $1.3 \%$ of the population regarded weak stream (5) as the most troublesome symptom. The other symptoms fall in-between these two.

\section{DISCUSSION}

The IPSS index may not be specific for BPH. However, it has been validated and is sensitive enough to be used in the screening for, evaluation of symptoms of, and selection of treatment for $\mathrm{BPH}^{5}$. This informed our confidence in the use of the IPSS index as a screening tool for BPH in this work.

The study revealed an age-dependent increase in the severity of symptoms of BPH as seen in
Table 1. This finding agrees with Eaton ${ }^{1}$ who wrote that the only clearly defined risk factors for $\mathrm{BPH}$ are age and circulating androgens. Voiding symptoms were regarded as the most troublesome symptoms with increasing age (Table 5) while the percentage of those who regarded storage symptoms as the most troublesome dropped with increasing age. However, for all the age ranges, more than half of the respondents regarded storage symptoms as the most troublesome. In all cases, nocturia was regarded as the most troublesome symptom, followed by urgency (both of them being storage symptoms). This underscores the effect of storage symptoms on the quality of life of men in Nsukka.

As much as $81.08 \%$ of the studied population felt satisfied with their quality of life, while $18.91 \%$ were not entirely satisfied. Interestingly, the percentage of those who felt that voiding symptoms as against storage symptoms were the most troublesome symptoms remained about the same for those who were satisfied or not satisfied with their quality of life (Table 4). Irrespective of the subjects' quality of life rating, $23.3 \%$ or less of the population of each group regarded voiding symptoms as the most troublesome symptoms while $76.7 \%$ or more felt storage symptoms were the most troublesome symptoms. However, the fact that 43 people who were dissatisfied with their quality of life identified only urgency, straining and nocturia as the most troublesome symptoms may point to these three symptoms as being chiefly responsible for poor quality of life. These findings agree with $\mathrm{Scarpa}^{2}$ that storage symptoms are the most bothersome symptoms and have a greater impact on the patient's quality of life. The quality of life of respondents was also seen to decrease with increasing age (Table 3) and by extension, with increasing severity of symptoms.

The prevalence of BPH in the studied population may be safely put at $25.35 \%$ (the percentage of those with symptoms other than mild symptoms). This is in the neighbourhood of what has been reported elsewhere. Garraway et al. ${ }^{8}$ using a smaller sample size, but employing ultrasonography in addition to the urinary 
symptom questionnaire, in the United Kingdom, reported a prevalence of $25.3 \%$ for $\mathrm{BPH}$. Chicharo-Molero et al. ${ }^{9}$ using the IPSS questionnaire reported a prevalence of $24.94 \%$ in Spain. Verhamme et al. ${ }^{7}$, working on the TRIUMPH project, however reported a prevalence of $10.3 \%$ for $\mathrm{BPH}$.

The sad implication of this is that we may have a BPH crisis situation akin to that in the developed world. Regrettably, awareness of this situation is poor. We therefore recommend that government, non-governmental organizations, academic and research institutions should aggressively embark upon mass screening and public enlightenment campaigns, coupled with research into $\mathrm{BPH}$ and other disorders of the prostate. The drastic effect of $\mathrm{BPH}$ on the quality of life of men who constitute the bulk of our workforce has adverse economic implications and cannot be toyed with.

It is hoped that this work would help alert our adult male population on the need to go for early and routine screening for prostate disorders (say from age 50). Early detection of BPH makes management easy and lowers the impact $\mathrm{BPH}$ has on the QOL of the patient. This would ensure a reduction in the loss of manpower to $\mathrm{BPH}$ and the attendant low QOL and increase productivity and mass development that the society dearly needs and eagerly seeks.

\section{REFERENCES}

1.Eaton, C. L. (2003) Aetiology and Pathogenesis of Benign Prostatic Hyperplasia.Curr Opin Uro. 13:7-10.

2.Scarpa, R. M. (2001) Lower Urinary Tract Symptoms (LUTS): What Are the Implications for the Patients? Eur. Urol. 40:12-20.

3.Welch, G., Kawachi, I. and Barry, M. J. (1998) Distinction Between Symptoms of Voiding and Filling in Benign Prostatic Hyperplasia: Findings from Health
Professionals Follow-Up Study. Urology 113:161-163.

4.Barry, M. J., Fowler, F. J. Jr., O'Leary, M. P., Bruskeuritz, R. C., Holtgrewe, H. L., Mebust, W. K. and Cockett, A. T. (1992) (The Measurement Committee of the American Urological Association). The American Urological Association Symptom Index for Benign Prostatic Hyperplasia. Urol. 148:1549-1564.

5.Barry, M. J. (2001) Evaluation of Symptoms and Quality of Life in Men with Benign Proatatic Hyperplasia. Urology 58:25-32.

6.Tsukamoto, T., Kumamoto, Y., Masumori, N., Miyake, H., Rhodes, T., Girman, C. J., Guess, H. A., Jacobsen, S. J., and Lieber, M. M. (1995) Prevalence of Prostatism in Japanese Men in a Community-based Study with Comparison to a Similar American Study. Journal of Urology 154:391-395.

7.Verhamme K. M., Dieleman J. P., Bleumink G. S., van der Lei J., Sturkenboom M. C., Artibani W., Begaud B., Berges R., Borkowski A., Chappel C. R., Costello A., Dobronski P., Farmer R. D., Jimenez Cruz F., Jonas U., MacRae K., Pientka L., Rutten F. F., van Schayck C. P., Speakman M. J., Tiellac P., Tubaro A., Vallencien G. and Vela Navarrete $R$. (2002) Incidence and Prevalence of Lower Urinary Tract Symptoms Suggestive of Benign Prostatic Hyperplasia in Primary Care-the Triumph Project. Eur Urol. 42:323-328

8.Garraway W. M., Collins G. N. and Lee R. J. (1991) High Prevalence of Benign Prostatic Hypertrophy in the Community. Lancet 24:469-471.

9.Chicharro-Molero J. A., Burgos-Rodriguez R., Sanchez-Cruz J. J., del RosalSamaniego J. M., Rodero-Carcia P., and Rodriguez-Vallejo J. M. (1998) Prevalence of Benign Prostatic Hyperplasia in Spanish Men 40 Years Old or Older. J. Urol. 159:878-82. 